\title{
Paeonol, a Major Compound of Moutan Cortex, Attenuates Cisplatin-Induced Nephrotoxicity in Mice
}

\author{
Hyojung Lee, Gihyun Lee, Hyunseong Kim, and Hyunsu Bae \\ Department of Physiology, College of Korean Medicine, Kyung Hee University, 1 Hoeki-Dong, Dongdaemoon-gu, \\ Seoul 130-701, Republic of Korea
}

Correspondence should be addressed to Hyunsu Bae; hbae@khu.ac.kr

Received 4 April 2013; Accepted 21 August 2013

Academic Editor: Seong-Gyu Ko

Copyright (C) 2013 Hyojung Lee et al. This is an open access article distributed under the Creative Commons Attribution License, which permits unrestricted use, distribution, and reproduction in any medium, provided the original work is properly cited.

Cisplatin is an effective chemotherapeutic agent that is used for the treatment of a variety of cancers; however, its nephrotoxicity limits the use of this drug. In the present study, we examined whether paeonol, a major compound of Moutan Cortex, has protective effects on cisplatin-induced acute renal failure in mice. To accomplish this, Balb/c mice (6 to 8 wk of age, weighing 20 to $25 \mathrm{~g}$ ) were administered, Moutan Cortex $(300 \mathrm{mg} / \mathrm{kg})$ or paeonol $(20 \mathrm{mg} / \mathrm{kg})$ once a day. At day 4, mice received cisplatin $(30,20, \mathrm{or} 10 \mathrm{mg} / \mathrm{kg})$ intraperitoneally. The paeonol-treated group showed marked attenuation of serum creatine and blood urea nitrogen levels as well as reduced levels of proinflammatory cytokines and nitric oxide when compared to the control group. In addition, the paeonol-treated group showed prolonged survival and marked attenuation of renal tissue injury. Taken together, these results demonstrated that paeonol can prevent the renal toxic effects of cisplatin.

\section{Introduction}

Cisplatin (cis-diamminedichloroplatinum (II)) is a platinum compound that has revolutionized the treatment of various solid organ tumors. Because of its high effects, cisplatin is used for the treatment of a variety of malignancies, including testicular, head and neck, ovarian, cervical, non-small-cell lung carcinoma and many other types of cancer $[1,2]$. However, nephrotoxicity, the most common adverse effect of cisplatin, often requires dose reduction or withdrawal of cisplatin [3]. Therefore, various studies have been investigated to reduce cisplatin-induced nephrotoxicity.

Cisplatin-induced nephrotoxicity is related to direct tubular toxicity, inflammation, vascular factors, and oxidative stress [2]. Oxidative stress and inflammation have been suggested as the major mechanisms in the pathogenesis of cisplatin-induced nephrotoxicity [4].

Moutan Cortex, the root bark of Paeonia suffruticosa Andrews, is widely used in traditional medicine to treat various diseases including atherosclerosis, infection, and inflammation [5]. Previous studies have revealed that the extracts of Moutan Cortex can inhibit nitric oxide and tumor necrosis factor-alpha (TNF- $\alpha$ ) in activated mouse peritoneal macrophages [5]. Further, our recent studies suggested that
Moutan Cortex exerted recovery effects on cisplatin-induced nephrotoxicity in vitro [6]. In the present study, we searched whether extract of Moutan Cortex could ameliorate cisplatininduced upregulation of serum creatinine and blood urea nitrogen (BUN) in vivo. After the confirmation that Moutan Cortex has the potential to attenuate the nephrotoxicity of cisplatin, we hypothesized that paeonol, a major phenolic component of Moutan Cortex, could attenuate cisplatininduced renal damage. Recent studies have suggested that paeonol has various biological activities such as antiaggregatory, antioxidant, anxiolytic-like, and anti-inflammatory functions [7-9]. Moreover, it has been shown that natural antioxidants and anti-inflammatory agents such as curcumin, licorice extract, and Zingiber officinale can attenuate cisplatininduced nephrotoxicity $[10,11]$. In this study, we investigated whether paeonol attenuates cisplatin-induced renal damage by inhibiting inflammation and nitrosative stress.

\section{Materials and Methods}

2.1. Animals. Balb/c male mice (6 to $8 \mathrm{wk}$ of age, weighing 20 to $25 \mathrm{~g}$ ) were purchased from Orient Bio (Seongnam, Republic of Korea). All mice were kept under pathogen-free 
conditions with air conditioning and a $12 \mathrm{~h}$ light/dark cycle and had free access to food and water during the experiments. The study was approved by the Animal Care and Use Committee of Kyung Hee University.

2.2. Materials. Moutan Cortex extract was purchased from Sun Ten (Sun Ten Pharmaceutical Co., Ltd., Taiwan) and dissolved in distilled water to give a final concentration of $10 \%(\mathrm{w} / \mathrm{v})$. The herb suspension supernatant (HSS) was then obtained by centrifugation at $300 \times \mathrm{g}$ for $10 \mathrm{~min}$ at $4^{\circ} \mathrm{C}$. Next, the HSS was passed through a sterile $0.20 \mu \mathrm{m}$ pore size filter unit (Sartorius AG, Germany). Paeonol was purchased from Wako (Wako Pure Chemical Industries, Ltd., Japan) and dissolved in phosphate buffered saline (PBS) at a concentration of $1 \mathrm{mg} / \mathrm{ml}$. Cisplatin (cis-diammineplatinum II dichloride; Sigma-Aldrich, St. Louis, MO) was dissolved in $0.9 \%$ saline at a concentration of $1 \mathrm{mg} / \mathrm{ml}$. Paeonol is one of many active main compounds contained in Moutan Cortex. Our previous study showed that $1 \mathrm{~g}$ of Moutan Cortex contained $0.46 \pm 0.01 \mathrm{mg}$ of paeonol. [5]

2.3. Experimental Protocol. Each group of mice was administered Moutan Cortex $(300 \mathrm{mg} / \mathrm{kg}$ body wt) or paeonol (20 mg/kg body wt) orally for three days. The control groups were only administered PBS. At day 4, the mice were given a single i.p. injection of either cisplatin $(30,20$, or $10 \mathrm{mg} / \mathrm{kg}$ body wt) or an equal volume of saline. Mice were sacrificed at 48 or $72 \mathrm{~h}$ after the cisplatin administration for further evaluation.

\subsection{Assessment of Renal Function and Histologic Examination.} Blood samples were obtained from mice $0,24,48$, and $72 \mathrm{~h}$ after cisplatin injection. Renal and liver function was assessed based on the BUN, creatinine, alanine aminotransferase, and aspartate aminotransferase, which were measured using a Fuji DRI-CHEM 3500i (Fuji Photo Film, Ltd., Japan). Kidney tissue was fixed in $4 \%$ paraformaldehyde and then embedded in paraffin, after which it was cut into $5 \mu \mathrm{m}$ sections and stained with hematoxylin and eosin. Three pathologists who were blinded to the experiments scored the degree of tubular injury. Renal tubular injury was assessed using a semiquantitative score in which the percentage of cortical tubules showing epithelial necrosis was assigned a score of either 0 , none; $1,<10 \% ; 2,10-25 \% ; 3,25-75 \%$; or 4 , $>75 \%[12]$.

2.5. Kidney Proinflammatory Cytokines. To examine the proinflammatory cytokines after cisplatin administration, the levels of TNF- $\alpha$ and IL- $1 \beta$ were measured in the kidneys by enzyme linked immunosorbent assay (ELISA; BD Biosciences, USA). Briefly, snap-frozen kidney tissue was homogenized in a PRO-PREP protein extraction solution (iNtRON Biotechnology, Inc., Korea), after which it was incubated for 20 minutes in ice and then centrifuged at $13,000 \mathrm{rpm}\left(4^{\circ} \mathrm{C}\right)$ for 15 minutes. The supernatant was then used for kidney proinflammatory cytokine detection, which was accomplished using a BCA ${ }^{\mathrm{TH}}$ Protein Assay Kit (Thermo
Scientific, USA). The protein levels of cytokines were corrected for the total amount of protein, and the results were expressed as $\mathrm{pg} / \mathrm{mg}$.

2.6. Nitrite Estimation. Tissue nitrite was estimated using Greiss reagent and served as an indicator of $\mathrm{NO}$ production. Briefly, $500 \mu \mathrm{L}$ of Greiss reagent (1:1 solution of $1 \%$ sulfanilamide in $5 \%$ phosphoric acid and $0.1 \%$ naphthylamine diamine dihydrochloric acid in water) was added to $100 \mu \mathrm{L}$ of kidney homogenates. The nitrite concentration was calculated using a standard curve for sodium nitrite. Nitrite levels were expressed as $\mu \mathrm{M} / \mathrm{mg}$.

2.7. Statistical Analysis. Statistical analysis of the data was conducted using Prism 4.02 software (GraphicPad Software Inc., USA). All data are presented as the means \pm S.E.M. The significance of differences between the experimental groups and the control was assessed by a Student's $t$-test. KaplanMeier analysis was used for the mouse survival analyses. For multiple comparison, two-way ANOVA was conducted. A $P$ value $<0.05$ was taken to indicate significance.

\section{Results}

To ascertain whether paeonol could attenuate cisplatininduced renal damage, we analyzed survival rate, serum creatinine, BUN, histologic change, proinflammatory cytokines, and nitrite production in cisplatin-injected mice.

3.1. Effects of Moutan Cortex on Cisplatin-Induced Renal Dysfunction. Both levels of the serum creatinine and BUN in cisplatin group were significantly increased at $48 \mathrm{~h}$ after cisplatin administration when compared with the saline group. The serum creatinine level in Moutan Cortex pretreatment group was significantly decreased at $48 \mathrm{~h}$ after cisplatin administration when compared with the cisplatin group (Figure 1(a)). In addition, BUN level was slightly reduced in Moutan Cortex pretreatment group at $48 \mathrm{~h}$ after cisplatin injection when compared with the cisplatin group (Figure 1(b)).

3.2. Effects of Paeonol on Kidney and Liver Dysfunction. Each group of mice was administered paeonol ( $20 \mathrm{mg} / \mathrm{kg}$ body wt) or saline via a single intraperitoneal injection for three days. At day 4, blood samples were obtained from mice to check the toxicity of paeonol. The dose of $20 \mathrm{mg} / \mathrm{kg}$ paeonol caused no harmful effects on kidney and liver dysfunction (Figure 2).

3.3. Effects of Paeonol on Survival after Cisplatin Administration. Both the paeonol-pretreated group and cisplatin group received three different doses of cisplatin $(30,20$, or $10 \mathrm{mg} / \mathrm{kg}$ body wt) and were followed up for $78 \mathrm{~h}$. At $78 \mathrm{~h}$ after injection of $30 \mathrm{mg} / \mathrm{kg}$ cisplatin, all of the cisplatin group mice had expired. However, the paeonol-pretreated mice had $28 \%$ survival. Moreover, at $72 \mathrm{~h}$ after $20 \mathrm{mg} / \mathrm{kg}$ of cisplatin administration, all of the paeonol-pretreated mice survived, but $42 \%$ of cisplatin group mice had expired. Taken together, 


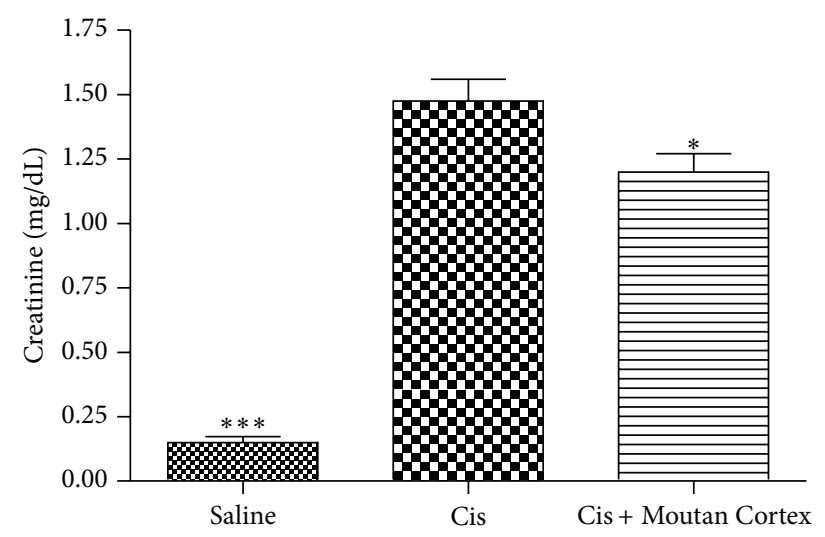

(a)

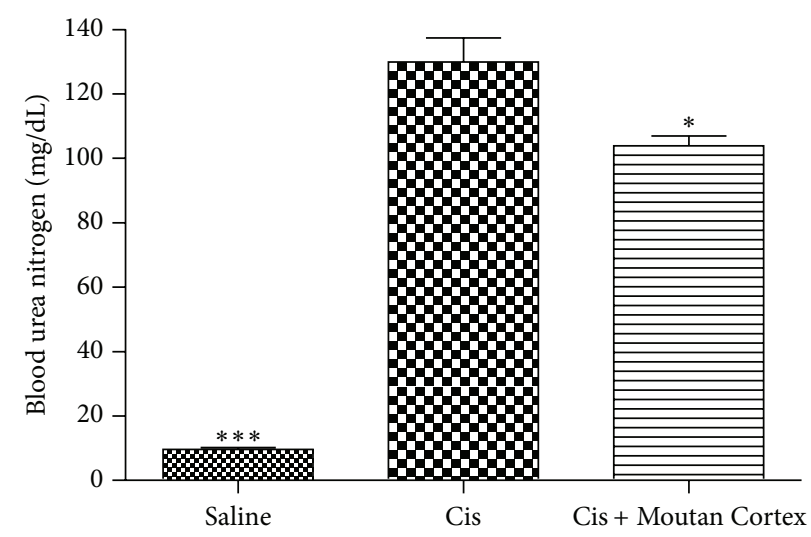

(b)

FIGURE 1: Effects of Moutan Cortex on creatinine and BUN production. All mice received a single dose of cisplatin intraperitoneally (30 mg/kg body wt). Serum creatinine (a) and BUN (b) were measured at $48 \mathrm{~h}$ after cisplatin injection. Saline: saline alone; Cis: cisplatin alone; Cis + Moutan Cortex: Moutan Cortex pretreatment and cisplatin treatment. Values shown are the mean \pm S.E.M. Data were analyzed by a Student's $t$-test $\left({ }^{*} P<0.05,{ }^{* * *} P<0.001\right.$ versus Cis; $\left.n=4\right)$.

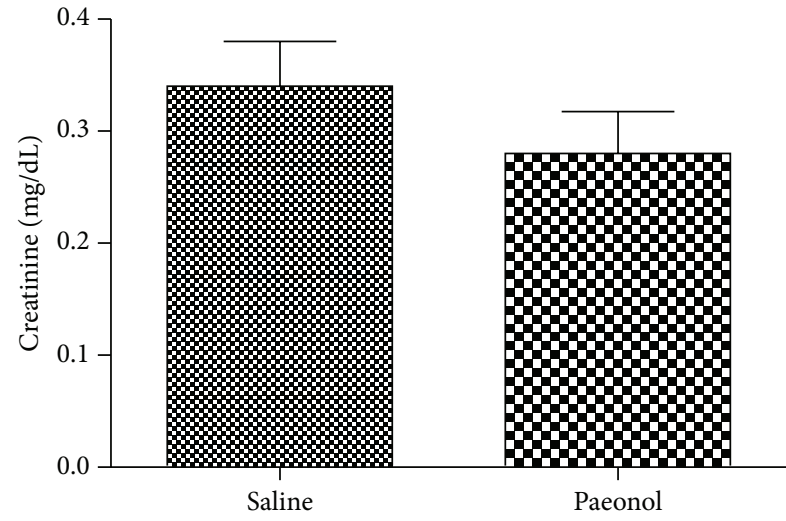

(a)

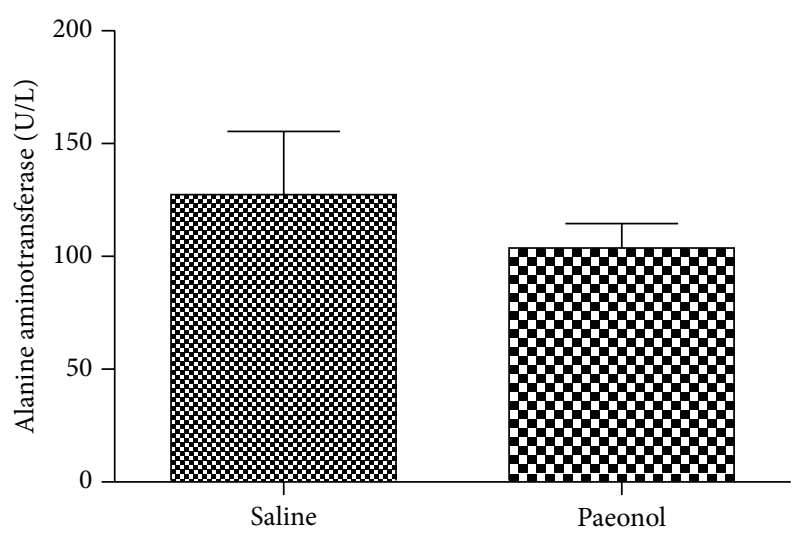

(c)

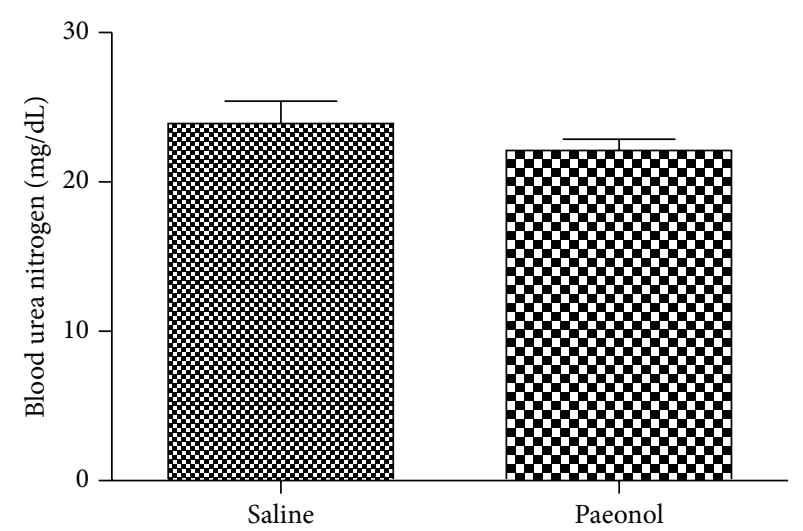

(b)

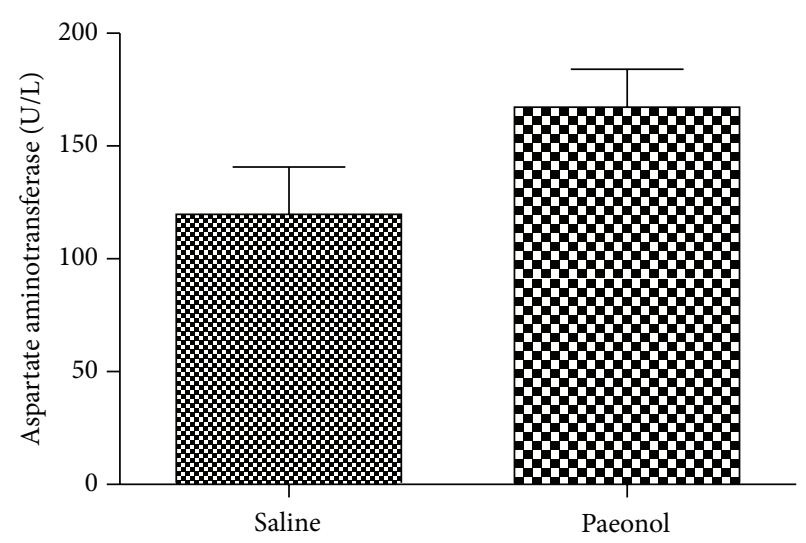

(d)

FIGURE 2: Effects of paeonol on kidney and liver dysfunction. Each group of mice was administered paeonol (20 mg/kg body wt) or saline via a single intraperitoneal injection for three days. At day 4, blood samples were obtained from mice to check the toxicity of paeonol. Serum creatinine (a), BUN (b), alanine aminotransferase (c), and aspartate aminotransferase (d) were measured. Values shown are the mean \pm S.E.M. Data were analyzed by a Student's $t$-test $(n=5)$. 


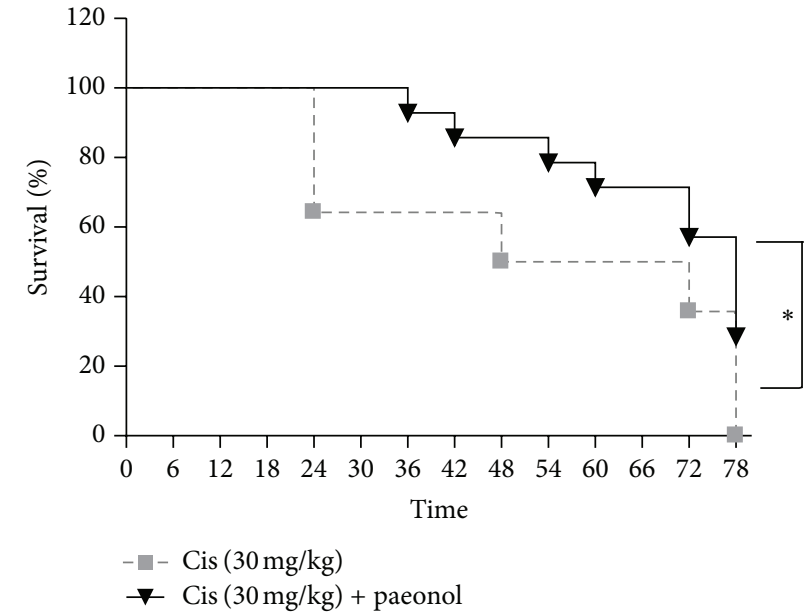

(a)

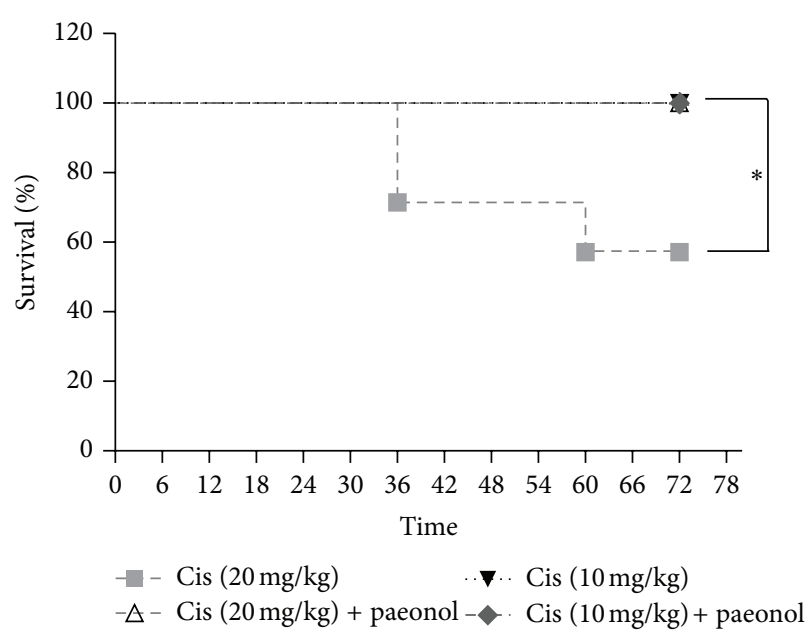

(b)

FIGURE 3: Survival in cisplatin-treated mice and paeonol-pretreated mice. (a) All mice received a single dose of cisplatin intraperitoneally $(30 \mathrm{mg} / \mathrm{kg}$ body wt) and were followed up to $78 \mathrm{~h}$. Compared with a 60 median survival time in cisplatin-treated group, paeonol-pretreated group had a 78 median survival time. Data were analyzed by a Kaplan-Meier test $\left({ }^{*} P<0.05\right.$ versus Cis; $\left.n=14\right)$. (b) Mice received cisplatin intraperitoneally ( 20 or $10 \mathrm{mg} / \mathrm{kg}$ body wt) and were followed up to $72 \mathrm{~h}$. Data were analyzed by a Kaplan-Meier test $\left({ }^{*} P<0.05\right.$ versus Cis; $n=7)$.

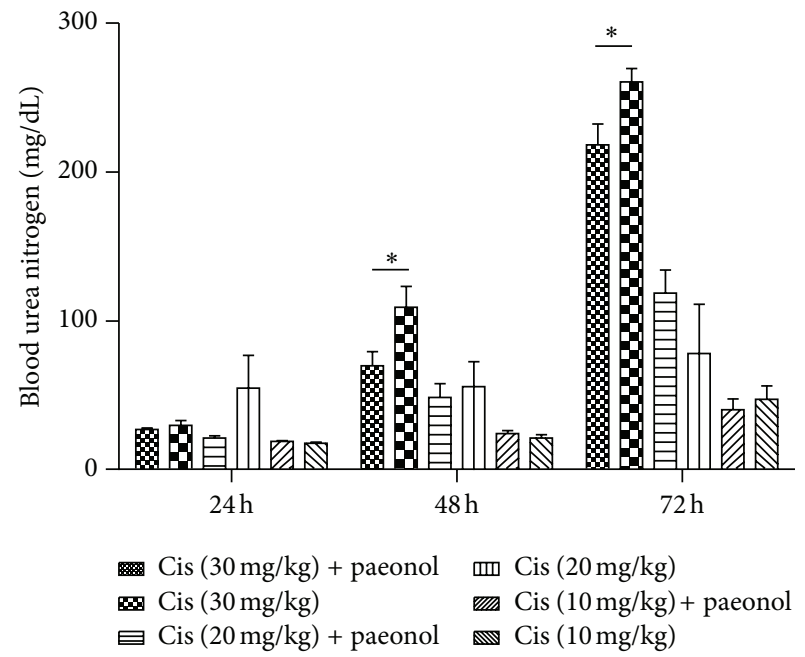

(a)

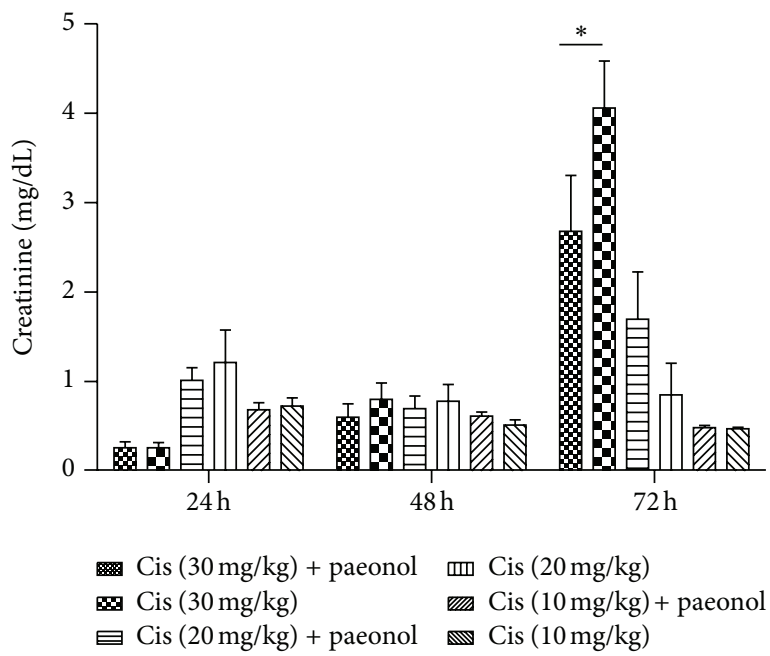

(b)

FIGURE 4: Effects of paeonol on cisplatin-induced renal dysfunction. Serum creatinine (a) and BUN (b) were measured at 24, 48, and $72 \mathrm{~h}$ after cisplatin injection $\left(30,20\right.$, or $10 \mathrm{mg} / \mathrm{kg}$ body wt). Data were analyzed by a two-way ANOVA test $\left({ }^{*} P<0.05 \mathrm{versus} \mathrm{Cis}(30 \mathrm{mg} / \mathrm{kg}) ; n=7\right)$.

these results show that pretreatment with paeonol improved survival after cisplatin administration in mice (Figure 3).

\subsection{Effects of Paeonol on Cisplatin-Induced Renal Dysfunction.}

The serum creatinine levels were significantly higher in the cisplatin group at $72 \mathrm{~h}$ after administration of $30 \mathrm{mg} / \mathrm{kg}$ of cisplatin when compared with the paeonol pretreatment group (Figure 4(a)). BUN level was also remarkably reduced in the paeonol pretreatment group at $48 \mathrm{~h}$ and $72 \mathrm{~h}$ after $30 \mathrm{mg} / \mathrm{kg}$ of cisplatin injection when compared with the group that only received cisplatin (Figure 4(b)). In $20 \mathrm{mg} / \mathrm{kg}$ of cisplatin-administered mice, creatinine and BUN levels were slightly decreased at $72 \mathrm{~h}$ by paeonol pretreatment. Besides, creatinine and BUN levels were not increased by $10 \mathrm{mg} / \mathrm{kg}$ of cisplatin treatment as previously reported $[13,14]$.

\subsection{Effects of Paeonol on Cisplatin-Induced Renal Injury.} The degree of renal tubular injury in the control- and paeonol-treated groups was observed at $72 \mathrm{~h}$ after cisplatin administration. Paeonol-treated groups showed slightly less renal injury than mice that received saline (Figure 5).

3.6. Effects of Paeonol on Cisplatin-Induced Proinflammatory Cytokines. To investigate the proinflammatory molecules 


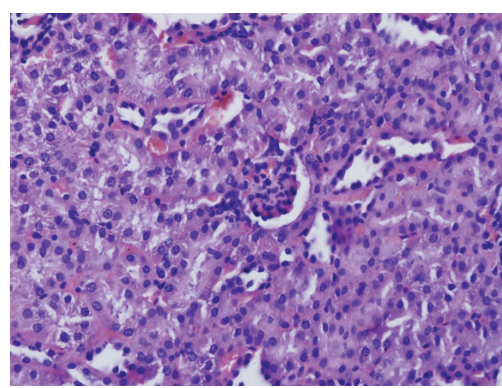

(a)

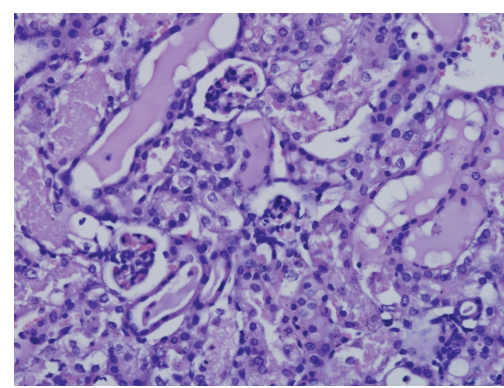

(b)

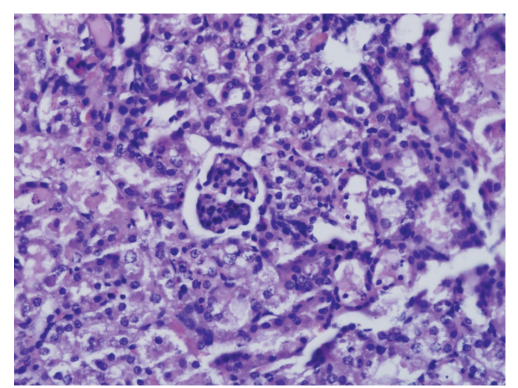

(c)

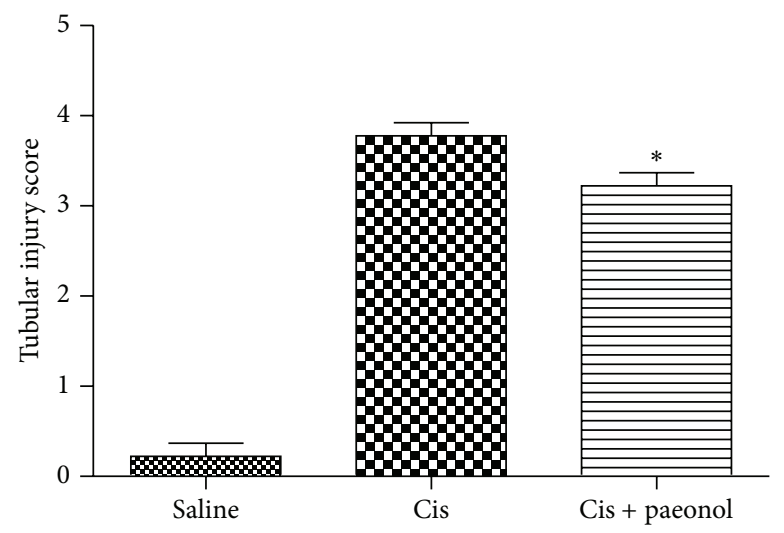

(d)

FIGURE 5: Effects of paeonol on renal histology in mice. Sections of kidney were stained with H\&E $72 \mathrm{~h}$ after cisplatin injection. Saline treatment alone (a); cisplatin treatment alone (b); paeonol and cisplatin treatment (c). Renal tubular injury was assessed using a semiquantitative score in which the percentage of cortical tubules showing epithelial necrosis was assigned a score of either 0 , none; $1,<10 \%$; 2, 10-25\%; 3, 25-75\%; or 4, >75\% (d). Saline: saline alone; Cis: cisplatin alone; Cis + paeonol: paeonol pretreatment and cisplatin treatment $\left({ }^{*} P<0.05\right.$ versus Cis, $\left.n=3\right)$.

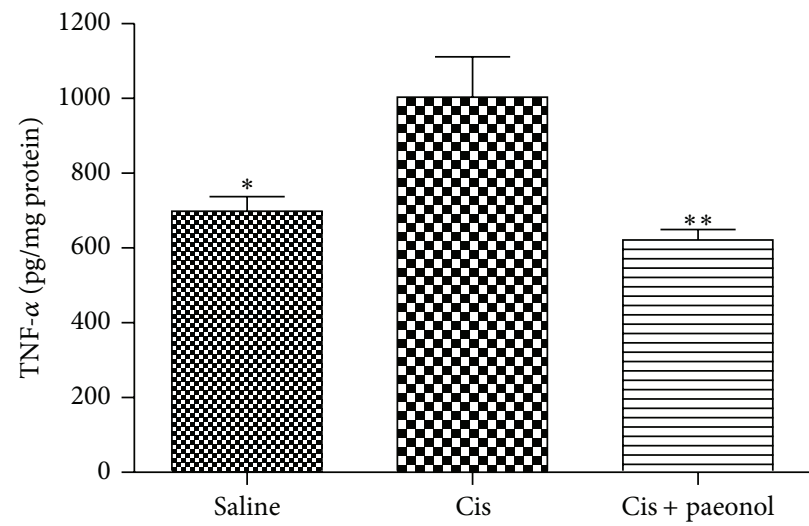

(a)

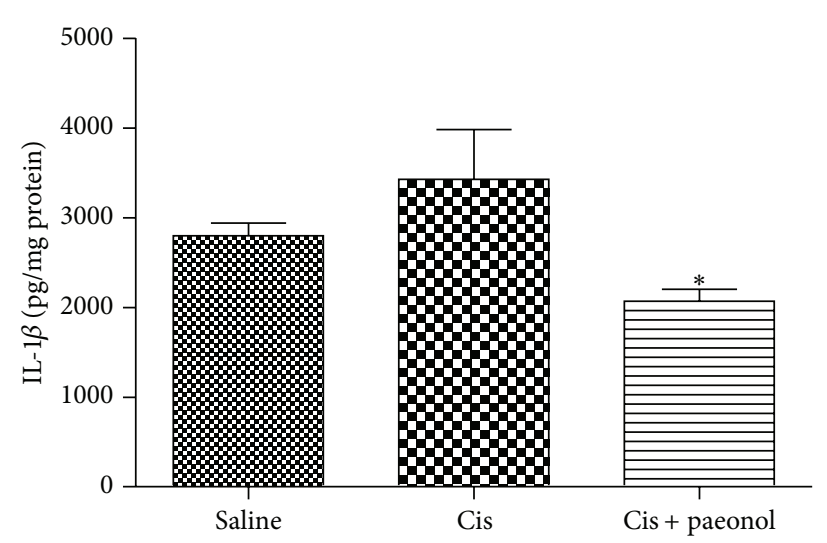

(b)

FIGURE 6: Effects of paeonol on cisplatin-induced proinflammatory cytokines. Kidneys were obtained at $72 \mathrm{~h}$ after cisplatin injection, and cytokine levels of TNF- $\alpha$ (a) and IL-1 $\beta$ (b) were determined by ELISA. Saline: saline alone; Cis: cisplatin alone; Cis + paeonol: paeonol pretreatment and cisplatin treatment. Values shown are the mean \pm S.E.M. Data were analyzed by a Student's $t$-test $\left({ }^{*} P<0.05,{ }^{* *} P<0.01\right.$ versus cis; $n=4$ for saline; $n=5$ for Cis or Cis + paeonol).

generated by cisplatin renal injury, the cytokine levels of TNF- $\alpha$ and IL-1 $\beta$ were measured at $72 \mathrm{~h}$ after cisplatin administration. Cisplatin-treated mice had significantly increased levels of TNF- $\alpha$ at $72 \mathrm{~h}$ after cisplatin injection, while paeonol pretreatment reduced expression of TNF- $\alpha$ in the kidney. IL-1 $\beta$ was increased by cisplatin treatment, but its increasing was not significant. However, paeonol significantly reduced IL- $1 \beta$ production by cisplatin treatment (Figure 6).

3.7. Effects of Paeonol on Cisplatin-Induced Nitric Oxide Production. Nitrite levels of kidney tissue homogenates were 


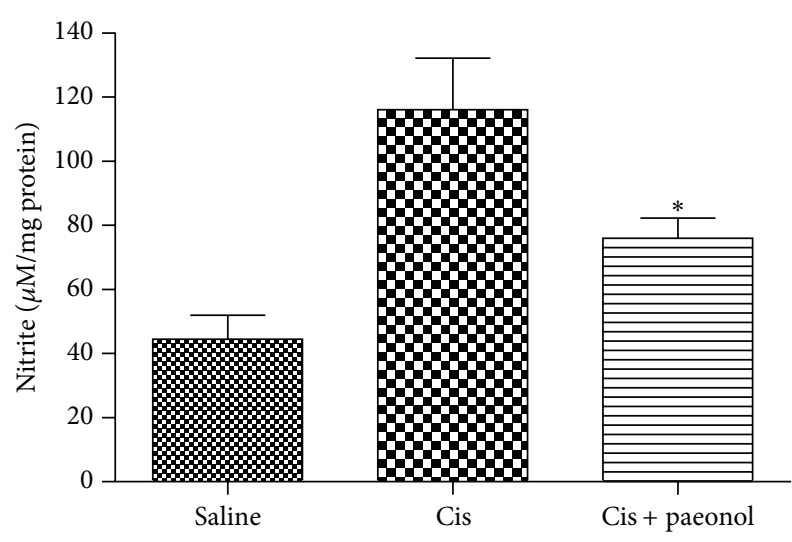

FIGURE 7: Effects of paeonol on cisplatin-induced nitric oxide production. Nitrate levels in kidney tissue were measured in control, cisplatin, and paeonol groups after a single dose of cisplatin (30 mg/kg body wt). Saline: saline alone; Cis: cisplatin alone; Cis + paeonol: paeonol pretreatment and cisplatin treatment. Values shown are the mean \pm S.E.M. Data were analyzed by a Student's $t$ test $\left({ }^{*} P<0.05\right.$ versus cis; $n=4$ for saline; $n=5$ for Cis or Cis + paeonol).

measured at $72 \mathrm{~h}$ after cisplatin injection. Nitrite levels of kidney tissue were significantly elevated by cisplatin administration. The nitrite production in kidney tissue was slightly attenuated in paeonol-pretreated group compared with control group (Figure 7).

\section{Discussion}

Nephrotoxicity is a major side effect that limits the use of cisplatin in many cancer patients. Indeed, many studies have documented that apoptosis/necrosis, reactive oxygen species, and inflammation play a major role in the pathogenesis of cisplatin-induced acute renal failure $[15,16]$. Therefore, various studies have been conducted to reduce the cisplatininduced nephrotoxicity. Recent studies have suggested that natural antioxidants and anti-inflammatory agents such as curcumin, licorice extract, and Zingiber officinale can attenuate cisplatin-induced nephrotoxicity [10,11]. Additionally, proinflammatory cytokines such as TNF- $\alpha$, IL-6, and IL$1 \beta$ have been shown to be associated with cisplatin-induced acute renal failure [17-19].

Moutan Cortex, the root bark of Paeonia suffruticosa Andrews, has been used extensively as a traditional medicine for treatment of various diseases such as atherosclerosis, infection, and inflammation. Previous studies have revealed that the extracts of Moutan Cortex can inhibit nitric oxide and TNF- $\alpha$ in activated mouse peritoneal macrophages [5].

A variety of compounds including paeonoside, paeonolide, apiopaeonoside, paeoniflorin, oxypaeoniflorin, benzoyloxypaeoniflorin, benzoylpaeoniflorin, paeonol, and sugars have been identified in Moutan Cortex [20]. Paeonol, a major phenolic component of Moutan Cortex, has various biological activities such as antiaggregatory, antioxidant, anxiolytic-like, and anti-inflammatory functions [8]. In this study, paeonol treatment significantly reduced the elevated levels of serum creatinine and BUN.

In addition, the role of proinflammatory cytokines in cisplatin-induced acute renal failure has been well documented $[17,18]$, and elevation of the proinflammatory cytokines TNF$\alpha$ and IL- $1 \beta$ as well as that of IL- 6 has been demonstrated in humans with acute renal failure [21]. Given that recent research has demonstrated that TNF- $\alpha$ plays a significant role in cisplatin-induced nephrotoxicity, we measured several proinflammatory cytokine levels in kidney tissue. We found that cisplatin administration increased the kidney tissue TNF- $\alpha$ level, whereas paeonol pretreatment led to a significant decrease in the TNF- $\alpha$ level. The increase in IL-1 $\beta$ level was also attenuated in paeonol-pretreated mice. Taken together, these results indicate that paeonol may reduce the production of TNF- $\alpha$ and IL- $1 \beta$ cytokines that is due to the direct anti-inflammatory effects of paeonol.

Nitric oxide (NO) is recognized as a mediator and regulator of inflammatory responses. NO is involved in host defense with cytotoxic properties against pathogenic microbes, but high levels of NO production in response to inflammatory stimuli can cause proinflammatory and destructive effects on host tissue [22]. Cisplatin treatment also causes significant increases in the activity of calcium-independent nitric oxide synthase (NOS) in rat kidney and liver, which leads to an increase in serum NO levels as well as tissue NO formation $[23,24]$. Jung et al. reported that the upregulation of oxide mNOS and peroxynitrite formation in cisplatin treatment are key events that influence the development of harmful parameters that occur during cisplatin-associated kidney failure [25]. It is well known that selective iNOS inhibition reduces cisplatin-induced nephrotoxicity and nitrosative stress [26]. In the present study, paeonol treatment prevented the increases in kidney tissue nitric oxide when compared with cisplatin-treated mice. Recently, paeonol treatment was found to inhibit iNOS protein expression induced by TNF$\alpha$ and iNOS mRNA expression in vitro $[8,27]$. Therefore, our results indicate that paeonol has the potential to reduce nitrosative stress in cisplatin-induced nephrotoxicity.

In conclusion, our study provides evidence that Moutan Cortex has a potential to attenuate nephrotoxicity of cisplatin and its major constituent paeonol can be a therapeutic intervention for cisplatin-induced renal injury. However, what molecular mechanisms are related to the protective effects of paeonol on kidney and the influences of Moutan Cortex and paeonol on the therapeutic efficacy of cisplatin should be further studied.

\section{Conflict of Interests}

The authors have declared that there is no conflict of interests.

\section{Acknowledgment}

This work was supported by the National Research Foundation of Korea (NRF) grant funded by the Korean government 
(Ministry of Education, Science and Technology, MEST) (No. 2011-0006220).

\section{References}

[1] D. Wang and S. J. Lippard, "Cellular processing of platinum anticancer drugs," Nature Reviews Drug Discovery, vol. 4, no. 4, pp. 307-320, 2005.

[2] N. Pabla and Z. Dong, "Cisplatin nephrotoxicity: mechanisms and renoprotective strategies," Kidney International, vol. 73, no. 9, pp. 994-1007, 2008.

[3] F. Ries and J. Klastersky, "Nephrotoxicity induced by cancer chemotherapy with special emphasis on cisplatin toxicity," American Journal of Kidney Diseases, vol. 8, no. 5, pp. 368-379, 1986.

[4] C. A. Davis, H. S. Nick, and A. Agarwal, "Manganese superoxide dismutase attenuates cisplatin-induced renal injury: importance of superoxide," Journal of the American Society of Nephrology, vol. 12, no. 12, pp. 2683-2690, 2001.

[5] H. S. Chung, M. Kang, C. Cho et al., "Inhibition of nitric oxide and tumor necrosis factor-alpha by moutan cortex in activated mouse peritoneal macrophages," Biological and Pharmaceutical Bulletin, vol. 30, no. 5, pp. 912-916, 2007.

[6] S. H. Sohn, H. Lee, J. Y. Nam et al., "Screening of herbal medicines for the recovery of cisplatin-induced nephrotoxicity," Environmental Toxicology and Pharmacology, vol. 28, no. 2, pp. 206-212, 2009.

[7] T. C. Chou, "Anti-inflammatory and analgesic effects of paeonol in carrageenan-evoked thermal hyperalgesia," British Journal of Pharmacology, vol. 139, no. 6, pp. 1146-1152, 2003.

[8] K. Ishiguro, T. Ando, O. Maeda et al., "Paeonol attenuates TNBS-induced colitis by inhibiting NF- $\kappa$ B and STAT1 transactivation," Toxicology and Applied Pharmacology, vol. 217, no. 1, pp. 35-42, 2006.

[9] J. M. Xiao, W. C. Si, J. W. Wen et al., "Anxiolytic-like effect of paeonol in mice," Pharmacology Biochemistry and Behavior, vol. 81, no. 3, pp. 683-687, 2005.

[10] T. A. Ajith, V. Nivitha, and S. Usha, "Zingiber officinale Roscoe alone and in combination with $\alpha$-tocopherol protect the kidney against cisplatin-induced acute renal failure," Food and Chemical Toxicology, vol. 45, no. 6, pp. 921-927, 2007.

[11] A. Kuhad, S. Pilkhwal, S. Sharma, N. Tirkey, and K. Chopra, "Effect of curcumin on inflammation and oxidative stress in cisplatin-induced experimental nephrotoxicity," Journal of Agricultural and Food Chemistry, vol. 55, no. 25, pp.10150-10155, 2007.

[12] H. Lee, D. Nho, H. S. Chung et al., " $\mathrm{CD} 4^{+} \mathrm{CD} 25^{+}$regulatory $\mathrm{T}$ cells attenuate cisplatin-induced nephrotoxicity in mice," Kidney International, vol. 78, no. 11, pp. 1100-1109, 2010.

[13] D. Z. Hsu, K. T. Chen, T. H. Lin, Y. H. Li, and M. Y. Liu, "Sesame oil attenuates cisplatin-induced hepatic and renal injuries by inhibiting nitric oxide-associated lipid peroxidation in mice," Shock, vol. 27, no. 2, pp. 199-204, 2007.

[14] E. A. Leite, C. D. S. Giuberti, A. J. A. Wainstein et al., "Acute toxicity of long-circulating and $\mathrm{pH}$-sensitive liposomes containing cisplatin in mice after intraperitoneal administration," Life Sciences, vol. 84, no. 19-20, pp. 641-649, 2009.

[15] A. H. Lau, "Apoptosis induced by cisplatin nephrotoxic injury," Kidney International, vol. 56, no. 4, pp. 1295-1298, 1999.

[16] H. Matsushima, K. Yonemura, K. Ohishi, and A. Hishida, "The role of oxygen free radicals in cisplatin-induced acute renal failure in rats," Journal of Laboratory and Clinical Medicine, vol. 131, no. 6, pp. 518-526, 1998.

[17] S. Faubel, E. C. Lewis, L. Reznikov et al., "Cisplatin-induced acute renal failure is associated with an increase in the cytokines interleukin (IL)-1 $\beta$, IL-18, IL-6, and neutrophil infiltration in the kidney," Journal of Pharmacology and Experimental Therapeutics, vol. 322, no. 1, pp. 8-15, 2007.

[18] G. Ramesh and W. B. Reeves, "TNF- $\alpha$ mediates chemokine and cytokine expression and renal injury in cisplatin nephrotoxicity," Journal of Clinical Investigation, vol. 110, no. 6, pp. 835-842, 2002.

[19] G. Ramesh and W. B. Reeves, "Inflammatory cytokines in acute renal failure," Kidney International, vol. 66, no. 91, supplement, pp. S56-S61, 2004.

[20] G. Chen, L. Zhang, and Y. Zhu, "Determination of glycosides and sugars in moutan cortex by capillary electrophoresis with electrochemical detection," Journal of Pharmaceutical and Biomedical Analysis, vol. 41, no. 1, pp. 129-134, 2006.

[21] E. M. Simmons, J. Himmelfarb, M. T. Sezer et al., "Plasma cytokine levels predict mortality in patients with acute renal failure," Kidney International, vol. 65, no. 4, pp. 1357-1365, 2004.

[22] R. Korhonen, A. Lahti, H. Kankaanranta, and E. Moilanen, "Nitric oxide production and signaling in inflammation," Current Drug Targets, vol. 4, no. 4, pp. 471-479, 2005.

[23] A. Kuhad, N. Tirkey, S. Pilkhwal, and K. Chopra, "6-gingerol prevents cisplatin-induced acute renal failure in rats," Biofactors, vol. 26, no. 3, pp. 189-200, 2006.

[24] R. C. Srivastava, A. Farookh, N. Ahmad, M. Misra, S. K. Hasan, and M. M. Husain, "Evidence for the involvement of nitric oxide in cisplatin-induced toxicity in rats," Biometals, vol. 9, no. 2, pp. 139-142, 1996.

[25] M. Jung, G. Hotter, J. L. Viñas, and A. Sola, “Cisplatin upregulates mitochondrial nitric oxide synthase and peroxynitrite formation to promote renal injury," Toxicology and Applied Pharmacology, vol. 234, no. 2, pp. 236-246, 2009.

[26] Y. I. Chirino, J. Trujillo, D. J. Sánchez-González et al., "Selective iNOS inhibition reduces renal damage induced by cisplatin," Toxicology Letters, vol. 176, no. 1, pp. 48-57, 2008.

[27] H. S. Chae, O. H. Kang, Y. S. Lee et al., "Inhibition of LPSinduced iNOS, COX-2 and inflammatory mediator expression by paeonol through the MAPKs inactivation in RAW 264.7 cells," American Journal of Chinese Medicine, vol. 37, no. 1, pp. 181-194, 2009. 


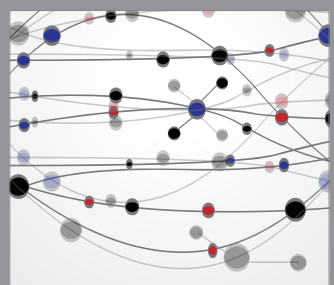

The Scientific World Journal
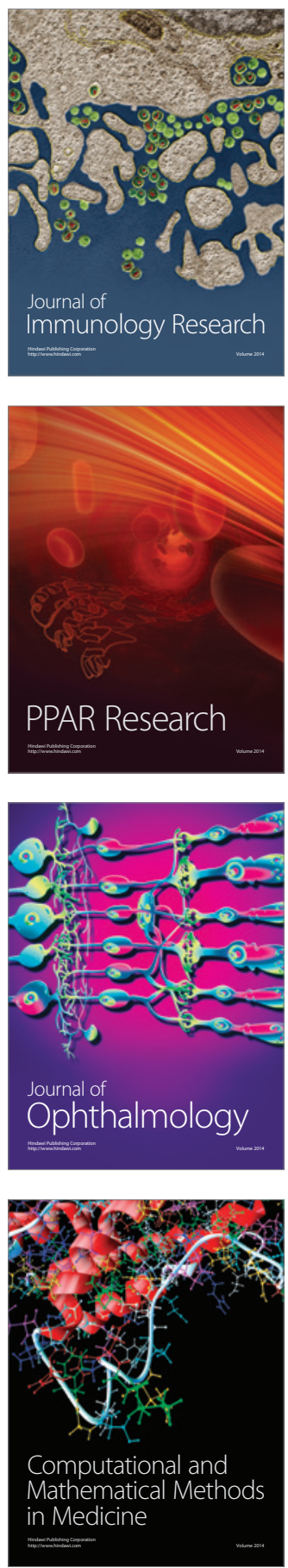

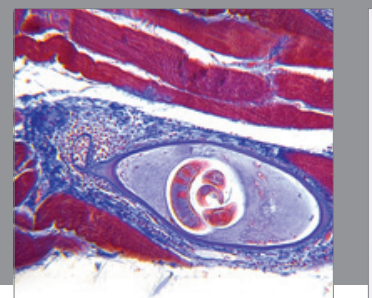

Gastroenterology

Research and Practice
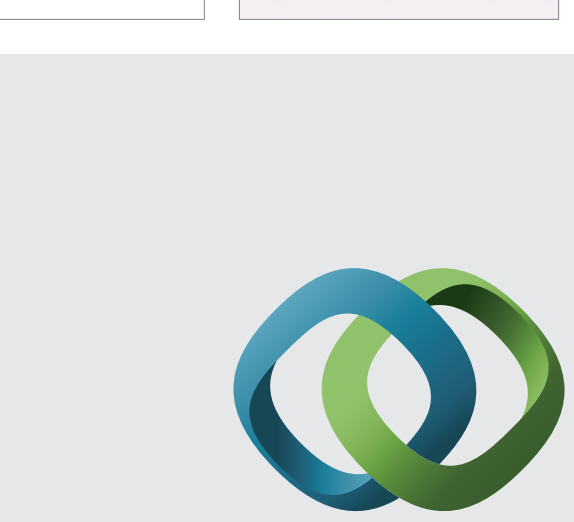

\section{Hindawi}

Submit your manuscripts at

http://www.hindawi.com
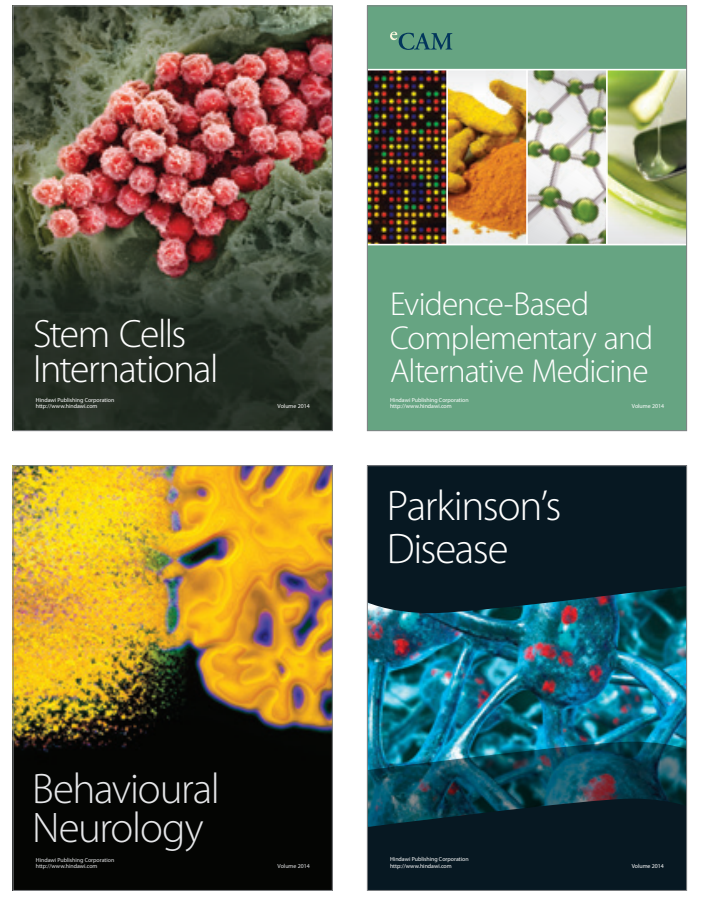
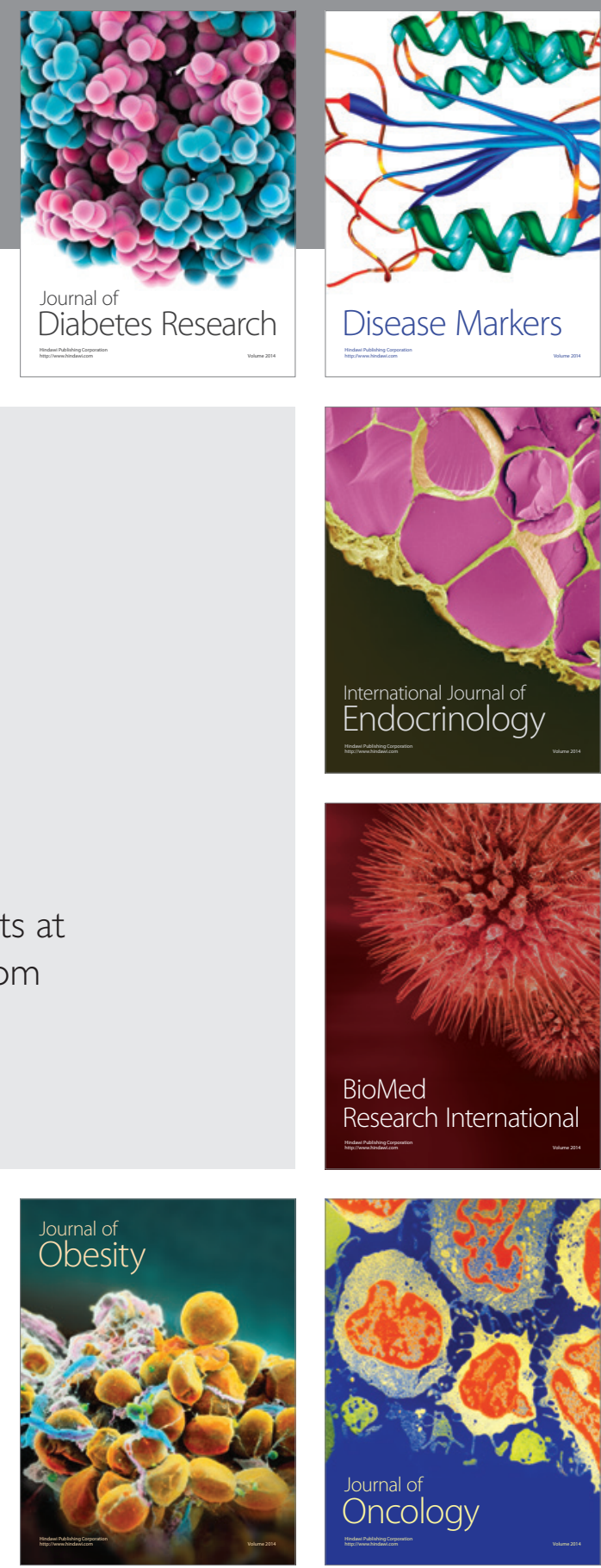

Disease Markers
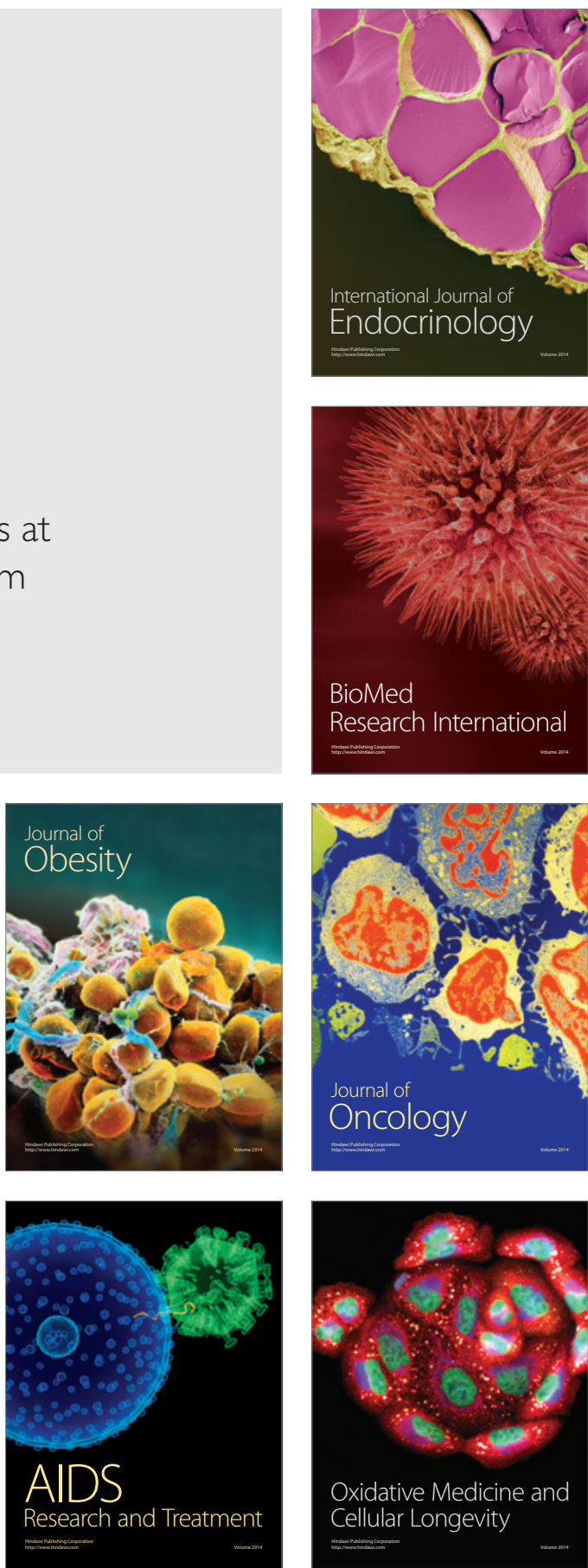